\title{
VSH, an Efficient and Provable Collision-Resistant Hash Function
}

\author{
Scott Contini ${ }^{1}$, Arjen K. Lenstra ${ }^{2}$, and Ron Steinfeld ${ }^{1}$ \\ ${ }^{1}$ Department of Computing, Macquarie University, NSW 2109, Australia \\ ${ }^{2}$ EPFL IC LACAL, INJ 330, Station 14, 1015-Lausanne, Switzerland
}

\begin{abstract}
We introduce VSH, very smooth hash, a new $S$-bit hash function that is provably collision-resistant assuming the hardness of finding nontrivial modular square roots of very smooth numbers modulo an $S$ bit composite. By very smooth, we mean that the smoothness bound is some fixed polynomial function of $S$. We argue that finding collisions for VSH has the same asymptotic complexity as factoring using the Number Field Sieve factoring algorithm, i.e., subexponential in $S$.

VSH is theoretically pleasing because it requires just a single multiplication modulo the $S$-bit composite per $\Omega(S)$ message-bits (as opposed to $O(\log S)$ message-bits for previous provably secure hashes). It is relatively practical. A preliminary implementation on a $1 \mathrm{GHz}$ Pentium III processor that achieves collision resistance at least equivalent to the difficulty of factoring a 1024-bit RSA modulus, runs at 1.1 MegaByte per second, with a moderate slowdown to $0.7 \mathrm{MB} / \mathrm{s}$ for 2048 -bit RSA security.

VSH can be used to build a fast, provably secure randomised trapdoor hash function, which can be applied to speed up provably secure signature schemes (such as Cramer-Shoup) and designated-verifier signatures.
\end{abstract}

Keywords: hashing, provable reducibility, integer factoring.

\section{Introduction}

Current collision-resistant hash algorithms that have provable security reductions are too inefficient to be used in practice. One example [17,20] that is provably reducible from integer factorisation is of the form $x^{m} \bmod n$ where $m$ is the message, $n$ a supposedly hard to factor composite, and $x$ is some prespecified base value. A collision $x^{m} \equiv x^{m^{\prime}} \bmod n$ reveals a multiple $m-m^{\prime}$ of the order of $x$ (which in itself divides $\phi(n)$ ). Such information can be used to factor $n$ in polynomial time assuming certain properties of $x$.

The above algorithm is quite inefficient because it requires on average 1.5 multiplications modulo $n$ per message-bit. Improved provable algorithms exist [7] which require a multiplication per $O(\log \log n)$ message-bits, but beyond that it seems that so far all attempts to gain efficiency came at the cost of losing provability (see also [1]). We propose a hash algorithm that uses a single multiplication per $\Omega(\log n)$ message-bits. It uses RSA-type arithmetic, obviating the need for completely separate hash function code such as SHA-1. Our algorithm may therefore be useful in embedded environments where code space is limited.

The original version of this chapter was revised: The copyright line was incorrect. This has been corrected. The Erratum to this chapter is available at DOI: 10.1007/978-3-540-34547-3_36

S. Vaudenay (Ed.): EUROCRYPT 2006, LNCS 4004, pp. 165-182, 2006.

(C) Springer-Verlag Berlin Heidelberg 2006 
We say that an integer is very smooth if its prime factors are bounded by $(\log n)^{c}$ for a fixed constant $c$. We use VSH, for very smooth hash, to refer to our new hash because finding a collision (i.e., strong collision resistance) for $\mathrm{VSH}$ is provably as difficult as finding a nontrivial modular square root of a very smooth number modulo $n$. We show that the latter problem, which we call VSSR, is connected to integer factorisation, and that it is reasonable to believe that VSSR is hard as well (until quantum computers are built). We emphasize that VSH is 'only' collision-resistant and not suitable as a substitute for a random oracle.

Given the factorisation of the VSH-modulus, collisions can be created (cf. trapdoor hashes in [20]). Therefore, for wide-spread application of a single VSHmodulus one has to rely on a trusted party to generate the modulus (and not to create collisions). Or one could use [2] to generate a modulus with knowledge of its factorisation shared among a group of authorities. For a one time computation the overhead may be acceptable. If each party would have it own VSH-modulus, the repudiation concerns are the same as those concerning regular RSA.

On the positive side, we show how VSH can be used to build a provably secure randomised trapdoor hash function which requires only about 4 modular multiplications to evaluate on fixed-length messages of length $k<\log _{2} n$ bits (compared to the fastest construction in [20], which requires about $k$ multiplications). Randomised trapdoor hash functions are used in signature schemes to achieve provable security against adaptive chosen message attack [20], and in designatedverifier signature schemes to achieve privacy $[11,21]$. Our function can replace the trapdoor function used in the Cramer-Shoup signature scheme [6], maintaining its provable security while speeding up verification time by about $50 \%$.

We also present a variant of VSH using a prime modulus $p$ (with no trapdoor), which has about the same efficiency and is provably collision-resistant assuming the hardness of finding discrete logarithms of very smooth numbers modulo $p$.

Related Work. Previous hash functions with collision resistance provably related to factoring have lower efficiency than VSH. The $x^{m} \bmod n$ function mentioned above appeared in $[17,20]$. A collision-resistant hash function based on a claw free permutation pair (where claw finding is provably as hard as factoring an RSA modulus) was proposed in [9] - this function requires 1 squaring per bit processed. In [7] the construction is generalised to use families of $r \geq 2$ claw free permutations, such that $\log _{2}(r)$ bits can be processed per permutation evaluation. Two factoring based constructions are presented, which require 2 multiplications per permutation evaluation. In the first construction the modulus $n$ has $1+\log _{2}(r)$ prime factors and thus becomes impractical already for small $\log _{2}(r)$. The second one uses a regular RSA modulus, but requires publishing $r$ random quadratic residues modulo $n$. This becomes prohibitive too for relatively small $\log _{2}(r)$; as a result the construction requires a multiplication modulo an $S$-bit RSA modulus $n$ per $O(\log S)$ message-bits while consuming polynomial space $(r=O(\operatorname{poly}(S)))$. The constructions in [1] are more efficient but are only provably collision-resistant assuming an underlying hash function is modeled as a random oracle (we make no such assumption). 
Section 2 introduces VSSR. VSH and its variants are presented in Section 3. Section 4 describes a VSH-based randomised trapdoor hash function which speeds up the Cramer-Shoup signature scheme. Section 5 concludes with implementation results.

\section{Security Definitions}

Notation. Throughout this paper, let $c>0$ be a fixed constant and let $n$ be a hard to factor $S$-bit composite for an integer $S>0$. The ring of integers modulo $n$ is denoted $\mathbf{Z}_{n}$, and its elements are represented by $\{0,1, \ldots, n-1\}$ or $\{-n+1,-n+2, \ldots, 0\}$. It will be clear from the context which representation is being used. The $i$ th prime is denoted $p_{i}: p_{1}=2, p_{2}=3, \ldots$, and $p_{0}=-1$. An integer is $p_{k}$-smooth if all its prime factors are $\leq p_{k}$. We use

$$
L[n, \alpha]=e^{(\alpha+o(1))(\log n)^{1 / 3}(\log \log n)^{2 / 3}}
$$

for constant $\alpha>0$ and $n \rightarrow \infty$, and where the logarithms are natural.

Definition 1. An integer $b$ is a very smooth quadratic residue modulo $n$ if the largest prime in b's factorisation is at most $(\log n)^{c}$ and there exists an integer $x$ such that $b \equiv x^{2} \bmod n$. The integer $x$ is said to be a modular square root of $b$.

Definition 2. An integer $x$ is said to be a trivial modular square root of an integer $b$ if $b=x^{2}$, i.e. $b$ is a perfect square and $x$ is the integer square root of $b$.

Trivial modular square roots have no relation to the modulus $n$. Such identities are easy to create, and therefore they are not allowed in the security reduction. A sufficient condition for a very smooth integer $b$ representing a quadratic residue not to have a trivial modular square root is having some prime $p$ such that $p$ divides $b$ but $p^{2}$ does not. Another sufficient condition is that $b$ is negative. Our new hardness assumption is that it is difficult to find a nontrivial modular square root of a very smooth quadratic residue modulo $n$. Before formulating our assumption, we give some relevant background on integer factorisation.

Background. General purpose integer factoring algorithms are used for the security evaluation of RSA, since they do not take advantage of properties of the factors. They all work by constructing nontrivial congruent squares modulo $n$ since such squares can be used to factor $n$ : if $x, y \in \mathbf{Z}$ are such that $x^{2} \equiv y^{2} \bmod n$ and $x \not \equiv \pm y \bmod n$, then $\operatorname{gcd}(x \pm y, n)$ are proper factors of $n$. To construct such $x, y$ a common strategy uses so-called relations. An example of a relation would be an identity of the form

$$
v^{2} \equiv \prod_{0 \leq i \leq u} p_{i}^{e_{i}(v)} \bmod n,
$$

where $u$ is some fixed integer, $v \in \mathbf{Z}$, and $\left(e_{i}(v)\right)_{i=0}^{u}$ is a $(u+1)$-dimensional integer vector. Given $u+1+t$ relations, at least $t$ linearly independent dependencies modulo 2 among the $u+1+t$ vectors $\left(e_{i}(v)\right)_{i=0}^{u}$ can be found using 
linear algebra. Each such dependency corresponds to a product of $v^{2}$-values that equals a product modulo $n$ of $p_{i}$ 's with all even exponents, and thus a solution to $x^{2} \equiv y^{2} \bmod n$. If $x \not \equiv \pm y \bmod n$, then it leads to a proper factor of $n$. A relation with all even exponents $e_{i}(v)$ leads to a pair $x, y$ right away, which has, in our experience with practical factoring algorithms, never happened unless $n$ is very small. It may safely be assumed that for each relation found at least one of the $e_{i}(v)$ 's is odd - actually most that are non-zero will be equal to 1 .

For any $u$, relations are easily computed if $n$ 's factorisation is known, since square roots modulo primes can be computed efficiently and the results can be assembled via the Chinese Remainder Theorem. If the factorisation is unknown, however, relations in practical factoring algorithms are found by a deterministic process that depends on the factoring algorithm used. It is sufficiently unpredictable that the resulting $x, y$ may be assumed to be random solutions to $x^{2} \equiv y^{2} \bmod n$, implying that the condition $x \not \equiv \pm y \bmod n$ holds for at least half of the dependencies. Despite the lack of a rigorous proof, this heuristic argument has not failed us yet. A few dependencies usually suffice to factor $n$.

The expected relation collection runtime is proportional to the product of $u$ (approximately the number of relations one needs) and the inverse of the smoothness probability of the numbers that one hopes to be $p_{u}$-smooth, since this probability is indicative for the efficiency of the collection process. For the fastest factoring algorithms published so far, the Number Field Sieve (NFS, cf. [13,5]), the overall expected runtime (including the linear algebra) is minimised - based on loose heuristic grounds - when, asymptotically for $n \rightarrow \infty$, u behaves as $L[n, 0.96 \ldots]$. For this $u$, the running time is $L[n, 1.923 \ldots]$, i.e., the square of $u$.

With the current state of the art of integer factorisation, one cannot expect that, for any value of $u$, a relation can be found faster than $L[n, 1.923 \ldots] / u$ on average, asymptotically for $n \rightarrow \infty$. For $u$-values much smaller than the optimum, the actual time to find a relation will be considerably larger (cf. remark below and [14]). For $u \approx(\log n)^{c}$, it is conservatively estimated that finding a relation requires runtime at least

$$
\frac{L[n, 1.923 \ldots]}{(\log n)^{c}}=L[n, 1.923 \ldots],
$$

asymptotically for $n \rightarrow \infty$, because the denominator gets absorbed in the numerator's $o(1)$. This observation that finding relations for very small $u$ (i.e., $u$ 's that are bounded by a polynomial function of $\log n$ ) can be expected to be asymptotically as hard as factoring $n$, is the basis for our new hardness assumption.

Before formulating it, we present two ways to use the hardness estimate $L[n, 1.923 \ldots] / u$ for small $u$ in practice. One way is to use the asymptotics and assume that finding a relation is as hard as factoring $n$. A more conservative approach incorporates the division by $u$ in the estimate. In theory this is a futile exercise because, as argued, a polynomially bounded $u$ disappears in the $o(1)$ for $n \rightarrow \infty$. In practice, however, $n$ does not go to infinity but actual values have to be dealt with. If $n^{\prime}$ is a hard to factor integer for which $\log n$ and $\log n^{\prime}$ are relatively close, then it is widely accepted that the ratio of the NFS-factoring runtimes for $n$ and $n^{\prime}$ approximates $L[n, 1.923 \ldots] / L\left[n^{\prime}, 1.923 \ldots\right]$ where the $o(1)$ 's 
are dropped. To assess the hardness estimate $L[n, 1.923 \ldots] / u$ for very small $u$, one therefore finds the least integer $S^{\prime}$ for which, after dropping the $o(1)$ 's,

$$
L\left[2^{S^{\prime}}, 1.923 \ldots\right] \geq \frac{L[n, 1.923 \ldots]}{u},
$$

and assumes that finding a relation for this $n$ and $u$ may be expected to be (at least) as hard as NFS-factoring a hard to factor $S^{\prime}$-bit integer. Note that $S^{\prime}$ will be less than $S$, the length of $n$. Examples of matching $S, S^{\prime}, u$ values are given in Section 5.

This factoring background provides the proper context for our new problem and its hardness assumption.

Definition 3. (VSSR: Very Smooth number nontrivial modular Square Root) Let $n$ be the product of two unknown primes of approximately the same size and let $k \leq(\log n)^{c}$. VSSR is the following problem: Given $n$, find $x \in Z_{n}^{*}$ such that $x^{2} \equiv \prod_{i=0}^{k} p_{i}^{e_{i}} \bmod n$ and at least one of $e_{0}, \ldots, e_{k}$ is odd.

VSSR Assumption. The VSSR assumption is that there is no probabilistic polynomial (in $\log n$ ) time algorithm which solves VSSR with non-negligible probability (the probability is taken over the random choice of the factors of $n$ and the random coins of the algorithm).

One can contrive moduli where VSSR is not difficult, such as if $n$ is very close to a perfect square. However, such examples occur with exponentially small probability assuming the factors of $n$ are chosen randomly, as required. According to proper security definitions [18], these examples do not even qualify as weak keys since the time-to-first-solution is slower than factoring, and therefore are not worthy of further consideration.

The VSSR Assumption is rather weak and useless in practice since it does not tell us for what size moduli VSSR would be sufficiently hard. This is similar to the situation in integer factorisation where the hardness assumption does not suffice to select secure modulus sizes. We therefore make an additional, stronger assumption that links the hardness of VSSR to the current state of the art in factoring. It is based on the conservative estimate for the difficulty of finding a relation for very small $u$ given above.

Computational VSSR Assumption. The computational VSSR assumption is that solving VSSR is as hard as factoring a hard to factor $S^{\prime}$-bit modulus, where $S^{\prime}$ is the least positive integer for which equation (1) holds (where, as in (1), the $o(1)$ 's in the $L[\ldots]$ 's are dropped).

Remark. For existing factoring algorithms, the relation collection runtime increases sharply for smoothness bounds that are too low, almost disastrously so if the bound is taken as absurdly low as in VSSR (cf. [14]). Therefore, the Computational VSSR Assumption is certainly overly conservative. Just assuming - as suggested above - that solving VSSR is as hard as factoring $n$ may be more accurate. Nevertheless, the runtime estimates for our new hash function will be based on the overly conservative Computational VSSR Assumption. 
Although our analysis is based on the average runtime to find a relation using the NFS, it is very conservative (i.e., leads to a large $n$ ) compared to a more direct analysis involving the relevant smoothness probability of squares modulo $n$. The latter would lead to a hardness estimate for finding even a single very smooth relation that is more similar to the runtime of the Quadratic Sieve integer factorisation algorithm, and thereby to much smaller 'secure' modulus sizes (obviously, unless $n$ 's factorisation is known or $n$ has a special form which it will not have when properly chosen). Thus, we feel more comfortable using our NFS-based approach.

\section{Very Smooth Hash Algorithm}

The basic version of VSH follows below. More efficient variants of VSH are discussed later in this section.

VSH Algorithm. Let $k$, the block length, be the largest integer such that $\prod_{i=1}^{k} p_{i}<n$. Let $m$ be an $\ell$-bit message to be hashed, consisting of bits $m_{1}, \ldots$, $m_{\ell}$, and assume $\ell<2^{k}$. To compute the hash of $m$ perform steps 1 through 5 :

1. Let $x_{0}=1$.

2. Let $\mathcal{L}=\left\lceil\frac{\ell}{k}\right\rceil$ (the number of blocks). Let $m_{i}=0$ for $\ell<i \leq \mathcal{L} k$ (padding).

3. Let $\ell=\sum_{i=1}^{k} \ell_{i} 2^{i-1}$ with $\ell_{i} \in\{0,1\}$ be the binary representation of the message length $\ell$ and define $m_{\mathcal{L} k+i}=\ell_{i}$ for $1 \leq i \leq k$.

4. For $j=0,1, \ldots, \mathcal{L}$ in succession compute

$$
x_{j+1}=x_{j}^{2} \times \prod_{i=1}^{k} p_{i}^{m_{j \cdot k+i}} \bmod n .
$$

\section{Return $x_{\mathcal{L}+1}$.}

Message Length. The message length does not need to be known in advance, which is useful for applications involving streaming data. In an earlier version which appeared on eprint [4], the message length was prepended, which may prove inconvenient and also required usage of $p_{k+1}$. If one uses the common method of appending a single 1 bit prior to zero-padding the final block, collisions can easily be created for the above version of VSH.

Compression Function $H$. VSH applies the compression function $H(x, m)$ : $\mathbf{Z}_{n}^{*} \times\{0,1\}^{k} \rightarrow \mathbf{Z}_{n}^{*}$ with $H(x, m)=x^{2} \prod_{i=1}^{k} p_{i}^{m_{i}} \bmod n$, and applies a variant of the Merkle-Damgård transformation $[15,8]$ to extend $H$ to arbitrarily long inputs. We comment on why this works in Section 3.1.

1024-Bit $n$. For 1024-bit $n$, the value for $k$ would be 131 . The requirement $\ell<2^{k}$ is therefore not a problem in any real application, and most of the bits $\ell_{i}$ will be zero. The Computational VSSR Assumption with $S=1024$ and $k=u=131$ leads to $S^{\prime}=840$. The security level obtained by VSH using 
1024-bit $n$ is therefore at least the security level obtained by 840 -bit RSA and, given recent hash developments, by SHA-1.

Efficiency. Because $\prod_{1 \leq i \leq K} p_{i}$ is asymptotically proportional to $e^{(1+o(1)) K \log K}$, for $K \rightarrow \infty$, the $k$ used in the basic version of VSH is proportional to $\frac{\log n}{\log \log n}$. It follows that the product $\prod_{i=1}^{k} p_{i}^{m_{j \cdot k+i}}$ can be computed in time $O\left((\log n)^{2}\right)$ using straightforward multiplication without modular reduction. Therefore the cost of each iteration is less than the cost of 3 modular multiplications. Since $k$ bits are processed per iteration, the basic version of VSH requires a single modular multiplication per $\Omega\left(\frac{\log n}{\log \log n}\right)$ message-bits, with a small constant in the $\Omega$.

Creating Collisions. With $e_{i}=\sum_{j=0}^{\mathcal{L}} m_{j \cdot k+i} 2^{\mathcal{L}-j}$ for $1 \leq i \leq k$, the value calculated by the VSH algorithm equals the multi-exponentiation $\prod_{i=1}^{k} p_{i}^{e_{i}} \bmod$ $n$. Given $\phi(n)$ and assuming large enough $\mathcal{L}$, collisions can be generated by replacing $e_{i}$ by $e_{i}+t_{i} \phi(n)$ for any set of $i$ 's with $1 \leq i \leq k$ and positive integers $t_{i}$ (see also VSH-DL below). Thus, parties that know $n$ 's factorisation can create collisions at will. But collisions of this sort immediately reveal $\phi(n)$ and thereby $n$ 's factorisation. Creating collisions that cannot immediately be used to factor $n$ is a harder problem, involving discrete logarithms of very smooth numbers.

To avoid repudiation concerns if VSH would be used 'globally' with the same modulus it would be advisable to generate $n$ using the method from [2]. On the other hand, it is conceivable - and may be desirable - to expand PKIs to allow one to choose one's own hash function, rather than using a 'fixed target' for all. In this setting, one cannot allow the owner of a VSH-modulus to claim he did not sign something by displaying a collision. Especially taking into consideration that the only easy way the user can create a collision would also reveal the factorisation of $n$, this would be analogous to somebody using RSA who anonymously posts the factorisation of their modulus in order to fraudulently claim that he did not sign something. Thus, in such a situation the VSH-modulus should be considered compromised and the user's certificate should be revoked.

Short Message Inversion. The VSH algorithm described above allows easy inversion of short and some sparse messages since there may be no wrap-around modulo $n$. The attacker first guesses the length, divides the hash modulo $n$ by the corresponding $\prod_{i=1}^{k} p_{i}^{m_{\mathcal{L} \cdot k+i}}$, and checks if the resulting value is very smooth. This type of invertibility may be undesirable for some applications, but others require just collision resistance (cf. below). See [16] for a related application.

A solution to this invertibility problem that does not affect our proof of security (cf. below) is to square the final output enough times to ensure wrap-around (no more than $\log _{2} \log _{2} n$ times). Other, more efficient solutions may be possible. Note that for all hash functions, the hash of extremely sparse or short messages can always be 'inverted' by trial and error.

Undesirable Properties. It is easy to find messages for which the hashes $h$ and $h^{\prime}$ satisfy $h=2 h^{\prime}$. Our solution to the invertibility problem addresses this issue as well. Other similar possibly undesirable properties can be constructed. We again 
emphasize that VSH is not intended to model a random oracle, and therefore cannot be blindly substituted as is into constructions that depend upon them (such as RSA signatures and some MAC constructions). We remind the reader that random oracles do not exist in the real world, and therefore relying on them too much is not recommended. On the other hand, entirely provable solutions do exist which require only collision resistance: for example, see Section 4.

Having stressed upfront in the last three remarks the disadvantages of VSH, we turn to its most attractive property, namely its provable collision resistance.

\subsection{Security Proof for VSH}

We prove that VSH is (strongly) collision-resistant. Using proper security notions [19], (strong) collision resistance also implies second preimage resistance.

Theorem 1. Finding a collision in VSH is as hard as solving VSSR (i.e., VSH is collision-resistant under the assumptions from Section 2).

Proof. We show that different colliding messages $m$ and $m^{\prime}$ lead to a solution of VSSR. Let $x_{\ldots}^{\prime}$ denote the $x \ldots$ values in the VSH algorithm applied to $m^{\prime}$ and let $\ell, \mathcal{L}$ and $\ell^{\prime}, \mathcal{L}^{\prime}$ be the bitlengths and number of blocks of $m$ and $m^{\prime}$, respectively. Since $m$ and $m^{\prime}$ collide, $m \neq m^{\prime}$ and $x_{\mathcal{L}+1}=x_{\mathcal{L}^{\prime}+1}^{\prime}$.

First consider the case of $\ell=\ell^{\prime}$. Let $m[j]$ denote $m$ 's $j$ th $k$-bit block, $m[j]=$ $\left(m_{j \cdot k+i}\right)_{i=1}^{k}$, and let $t \leq \mathcal{L}$ be the largest index such that $\left(x_{t}, m[t]\right) \neq\left(x_{t}^{\prime}, m^{\prime}[t]\right)$ but $\left(x_{j}, m[j]\right)=\left(x_{j}^{\prime}, m^{\prime}[j]\right)$ for $t<j \leq \mathcal{L}+1$. Then,

$$
\left(x_{t}\right)^{2} \times \prod_{i=1}^{k} p_{i}^{m_{t \cdot k+i}} \equiv\left(x_{t}^{\prime}\right)^{2} \times \prod_{i=1}^{k} p_{i}^{m_{t \cdot k+i}^{\prime}} \bmod n .
$$

Let $\Delta=\left\{i: m_{t \cdot k+i} \neq m_{t \cdot k+i}^{\prime}, 1 \leq i \leq k\right\}$ and $\Delta_{10}=\left\{i \in\{1, \ldots, k\}: m_{t \cdot k+i}=\right.$ 1 and $\left.m_{t \cdot k+i}^{\prime}=0\right\}$. Because all factors in Equation (2) are invertible modulo $n$, it is equivalent to

$$
\left[\left(x_{t} / x_{t}^{\prime}\right) \times \prod_{i \in \Delta_{10}} p_{i}\right]^{2} \equiv \prod_{i \in \Delta} p_{i} \bmod n .
$$

If $\Delta \neq \emptyset$, Equation (3) solves VSSR. If $\Delta=\emptyset$, then $\left(x_{t}\right)^{2} \equiv\left(x_{t}^{\prime}\right)^{2} \bmod n$ and $t \geq 1$ ( since $m \neq m^{\prime}$ and using the definition of $t$ ). With $x_{t} \not \equiv \pm x_{t}^{\prime} \bmod n$ VSSR can be solved by factoring $n$. If $x_{t} \equiv \pm x_{t}^{\prime} \bmod n$ then $x_{t} \equiv-x_{t}^{\prime} \bmod n$, since $\Delta=\emptyset$ implies (by definition of $t$ ) that $x_{t} \neq x_{t}^{\prime}$. But $x_{t} \equiv-x_{t}^{\prime} \bmod n$ leads to $\left(x_{t-1} / x_{t-1}^{\prime}\right)^{2}$ being congruent to -1 times a very smooth number and thus solves VSSR.

Now consider the case $\ell \neq \ell^{\prime}$. Since $x_{\mathcal{L}+1}=x_{\mathcal{L}^{\prime}+1}^{\prime}$, we have $\left(x_{\mathcal{L}} / x_{\mathcal{L}^{\prime}}^{\prime}\right)^{2} \equiv$ $\prod_{i=1}^{k} p_{i}^{\ell_{i}^{\prime}-\ell_{i}} \bmod n$. Since $\left|\ell_{i}^{\prime}-\ell_{i}\right|=1$ for at least one $i, \operatorname{VSSR}$ is solved using a transformation as in Equation (3).

Why Merkle-Damgård Works. VSH applies a variant of the Merkle-Damgård transformation $[15,8]$ to hash arbitrary length messages using the compression 
function $H: \mathbf{Z}_{n}^{*} \times\{0,1\}^{k} \rightarrow \mathbf{Z}_{n}^{*}$. The proof in [8] shows that a sufficient condition for a hash function to be collision-resistant is that its compression function $H$ is collision-resistant, i.e. it is hard to find any $(x, m) \neq\left(x^{\prime}, m^{\prime}\right)$ with $H(x, m)=$ $H\left(x^{\prime}, m^{\prime}\right)$. However, our compression function $H(x, m)=x^{2} \prod_{i=1}^{k} p_{i}^{m_{i}} \bmod n$ is not strictly collision-resistant $(H(-x \bmod n, m)=H(x, m))$, and yet we proved that $H$ is still sufficiently strong to make VSH collision-resistant. Therefore, one may ask whether we can strengthen the result in [8] to state explicitly the security properties of a compression function (which are weaker than full collision resistance) that our compression function satisfies and that are still sufficient in general to make the resulting hash function collision-resistant. Indeed, these conditions can be readily generalised from our proof of Theorem 1 , so we only state them here:

(1) Collision Resistance in Second input: It is hard to find $(x, m),\left(x^{\prime}, m^{\prime}\right) \in$ $\mathbf{Z}_{n}^{*} \times\{0,1\}^{k}$ with $m \neq m^{\prime}$ such that $H(x, m)=H\left(x^{\prime}, m^{\prime}\right)$.

(2) Preimage Resistance for a collision in first input: It is hard to find $(x, m) \neq$ $\left(x^{\prime}, m^{\prime}\right) \in \mathbf{Z}_{n}^{*} \times\{0,1\}^{k}$ and $m^{*} \in\{0,1\}^{k}$ such that $H\left(y, m^{*}\right)=H\left(y^{\prime}, m^{*}\right)$, where $y=H(x, m), y^{\prime}=H\left(x^{\prime}, m^{\prime}\right)$ and $y \neq y^{\prime}$.

The VSH compression function $H$ satisfies these properties, under the VSSR Assumption.

\subsection{Example: A Related Algorithm That Can Be Broken}

To emphasize the importance of the nontrivialness, consider a hash function that works similarly to VSH, except breaks the message into blocks $r_{1}, r_{2}, \ldots$ of $K>1$ bits and uses the compression function $x_{j+1}=x_{j}^{2} \times 2^{r_{j+1}} \bmod n$. Because $K>1$ collisions can simply be created. For example, for any $e$ with $0<e<2^{K-1}$ the message blocks $r_{1}=e$ and $r_{2}=2 e$ collide with $r_{1}^{\prime}=2 e$ and $r_{2}^{\prime}=0$. The colliding values are $\left(2^{e}\right)^{2} 2^{2 e}$ and $\left(2^{2 e}\right)^{2} 2^{0}$, but this does not lead to a solution of VSSR or a chance to factor $n$. Such trivial relations are useless, and the security of this hash algorithm is not based on a hard problem. The fix is to use the costlier compression function $x_{j+1}=x_{j}^{2^{K}} \times 2^{r_{j+1}}$, but that results in the same function $x^{m} \bmod n$ from $[17,20]$.

\subsection{Combining VSH and RSA}

Since the output length of VSH is the length of a secure RSA modulus (thus 1024-2048 bits), VSH seems quite suitable in practice for constructing 'hashthen-sign' RSA signatures for arbitrarily long messages. However, such a signature scheme must be designed carefully to ensure its security. To illustrate a naive insecure scheme, let $(n, e)$ be the signer's public RSA key, where the modulus $n$ is used for both signing and hashing. The signing function $\sigma:\{0,1\}^{*} \rightarrow \mathbf{Z}_{n}$ is $\sigma(m)=V S H_{n}(m)^{1 / e} \bmod n$, where $V S H_{n}:\{0,1\}^{*} \rightarrow \mathbf{Z}_{n}$ is VSH with modulus $n$. For a $k$-bit message $m=\left(m_{1}, \ldots, m_{k}\right) \in\{0,1\}^{k}$, the signature is thus $\sigma(m)=\left(\kappa \prod_{i=1}^{k} p_{i}^{2 m_{i}}\right)^{1 / e} \bmod n$, for a $\kappa$ that is the same for all $k$-bit messages. 
This scheme is insecure under the following chosen message attack. After obtaining signatures on three $k$-bit messages: $s_{0}=\sigma((0,0,0, \ldots, 0))=\kappa^{1 / e} \bmod$ $n, s_{1}=\sigma((1,0,0, \ldots, 0))=\left(\kappa p_{1}^{2}\right)^{1 / e} \bmod n$, and $s_{2}=\sigma((0,1,0, \ldots, 0))=$ $\left(\kappa p_{2}^{2}\right)^{1 / e} \bmod n$, the attacker easily computes the signature $\frac{s_{1} s_{2}}{s_{0}} \bmod n$ on the new $k$-bit forgery message $(1,1,0, \ldots, 0)$. It is easy to see that $k+1$ signatures on $k+1$ properly chosen messages suffice to sign any $k$-bit message.

To avoid such attacks, we suggest a more theoretically sound design approach for using VSH with 'hash-then-sign' RSA signatures that does not rely on any property of VSH beyond the collision resistance which it was designed to achieve:

Step 1. Let $\bar{n}$ be an $(S+1)$-bit RSA modulus, with $\bar{n}$ and the $S$-bit VSH modulus $n$ chosen independently at random. So, $\bar{n}>2^{S}$. Specify a one-to-one one-way encoding function $f:\{0,1\}^{S} \rightarrow\{0,1\}^{S}$, and define the short-message ( $S$-bit) RSA signature scheme with signing function $\sigma_{\bar{n}}(m)=(f(m))^{1 / e} \bmod$ $\bar{n}$. The function $f$ is chosen such that the short-message scheme with signing function $\sigma_{\bar{n}}$ is existentially unforgeable under chosen message attack. In the standard model no provable techniques are known to find $f$, but since $f$ is oneto-one, there are no collision resistance issues to consider when designing $f$.

Step 2. With $(\bar{n}, n, e)$ as the signer's public key, the signature scheme for signing arbitrary length messages is now constructed with signing function $\sigma_{\bar{n}, n}(m)=$ $\sigma_{\bar{n}}\left(V S H_{n}(m)\right)$. It is easy to prove that the scheme with signing function $\sigma_{\bar{n}, n}$ is existentially unforgeable under chosen message attack, assuming that the scheme with signing function $\sigma_{\bar{n}}$ is and that $V S H_{n}$ is collision-resistant. We emphasize that the proof no longer holds if $\bar{n}=n$ : in order to make the proof work in that case, one needs the stronger assumption that $V S H_{n}$ is collision-resistant even given access to a signing oracle $\sigma_{n}$. However, it is worth remarking that if the function $f$ is modeled as a random oracle, then the proof of security works (under the RSA and VSSR assumptions) even with a shared modulus $(\bar{n}=n)$.

\subsection{Variants of VSH}

Cubing Instead of Squaring. Let $H^{\prime}: \mathbf{Z}_{n}^{*} \times\{0,1\}^{k} \rightarrow \mathbf{Z}_{n}^{*}$ with $H^{\prime}(x, m)=$ $x^{3} \prod_{i=1}^{k} p_{i}^{m_{i}} \bmod n$ be a compression function that replaces the squaring in $H$ by a cubing. If $\operatorname{gcd}(3, \phi(n))=1$ then thanks to the injectivity of the RSA cubing map modulo $n$, the function $H^{\prime}$ is collision-resistant, assuming the difficulty of computing a modular cube root of a very smooth cube-free integer of the form $\prod_{i=1}^{k} p_{i}^{e_{i}} \neq 1$, where $e_{i} \in\{0,1,2\}$ for all $i$. This problem is related to RSA inversion, and is also conjectured to be hard. Although $H^{\prime}$ requires about 4 modular multiplications per $k$ message-bits (compared to 3 for $H$ ), it has the interesting property that $H^{\prime}$ itself is collision-resistant, while this is not quite the case for $H$ (because $\left.x^{2} \prod_{i} p_{i}^{m_{i}} \equiv(-x)^{2} \prod_{i} p_{i}^{m_{i}} \bmod n\right)$.

Increasing the Number of Small Primes. A speed-up is obtained by allowing the use of larger $k$ than the largest one for which $\prod_{i=1}^{k} p_{i}<n$. This does not affect the proof of security and reduction to VSSR, as long as $k$ is still polynomially bounded in $\log n$. The Computational VSSR Assumption implies that a larger modulus $n$ has to be used to maintain the same level of security. 
Furthermore, the intermediate products in Step 4 of the VSH algorithm may get larger than $n$ and may thus have to be reduced modulo $n$ every so often. Nevertheless, the resulting smaller $\mathcal{L}$ may outweigh these disadvantages.

Precomputing Products of Primes. An implementation speed-up may be obtained by precomputing products of primes. Let $b>1$ be a small integer, and assume that $k=\bar{k} b$ for some integer $\bar{k}$. For $i=1,2, \ldots, \bar{k}$ compute the $2^{b}$ products over all subsets of the set of $b$ primes $\left\{p_{(i-1) b+1}, p_{(i-1) b+2}, \ldots, p_{i b}\right\}$, resulting per $i$ in $2^{b}$ moderately sized values $v_{i, t}$ for $0 \leq t<2^{b}$. The $k$ messagebits per iteration of VSH are now split into $\bar{k}$ chunks $m[0], m[1], \ldots, m[\bar{k}-1]$ of $b$ bits each, interpreted as non-negative integers $<2^{b}$. The usual product is then calculated as $\prod_{i=1}^{\bar{k}} v_{i, m[i-1]}$. This has no effect on the number of iterations or the modulus size to be used to achieve a certain level of security.

Fast VSH. Redefining the above $v_{i, t}$ as $p_{(i-1) 2^{b}+t+1}$ and using $i=1,2, \ldots, k$ instead of $i=1,2, \ldots, \bar{k}$, the block length increases from $k$ to $b k$, the number of iterations is reduced from $\left\lceil\frac{\ell}{k}\right\rceil$ to $\left\lceil\frac{\ell}{b k}\right\rceil$, and the calculation in Step 4 of the VSH algorithm becomes

$$
x_{j+1}=x_{j}^{2} \times \prod_{i=1}^{k} p_{(i-1) 2^{b}+m[j b k+i-1]+1} \bmod n,
$$

where $m[r]$ is the $r$ th $b$-bit chunk of the message, with $0 \leq m[r]<2^{b}$. Because the number of small primes increases from $k$ to $k 2^{b}$, a larger modulus would, conservatively, have to be used to maintain the same level of security. But this change does not affect the proof of security and, as shown in the analysis below and the runtime examples in the final section, it is clearly advantageous.

Analysis of Fast VSH. Since $p_{(i-1) 2^{b}+m[j b k+i-1]+1} \leq p_{i 2^{b}}$, each intermediate product in the compression function for Fast VSH will be less than $n$ if $\prod_{i=1}^{k} p_{i 2^{b}}<n$. If $k$ is maximal such that $\prod_{i=1}^{(k+1) 2^{b}} p_{i} \leq(2 n)^{2^{b}}$, then

$$
\prod_{i=1}^{(k+1) 2^{b}} p_{i}=\prod_{t=1}^{2^{b}} \prod_{i=0}^{k} p_{i 2^{b}+t} \leq(2 n)^{2^{b}}
$$

so that $\prod_{i=0}^{k} p_{i 2^{b}+1} \leq 2 n$. With $p_{i 2^{b}}<p_{i 2^{b}+1}$ it follows that $\prod_{i=1}^{k} p_{i 2^{b}}<n$. Thus, for $(k+1) 2^{b}$ proportional to $\frac{2^{b} \log (2 n)}{\log \left(2^{b} \log (2 n)\right)}$ and $k$ to $\frac{\log (2 n)}{\log \left(2^{b} \log (2 n)\right)}-1$, the cost of Fast VSH is one modular multiplication per $\Omega(b k)$ message-bits, with $b k$ proportional to $\frac{b \log (2 n)}{\log \left(2^{b} \log (2 n)\right)}-b$. Selecting $2^{b}$ as any fixed positive power of $\log (2 n)$, it follows that $b k$ is proportional to $\log n$ and thus that Fast VSH requires a single modular multiplication per $\Omega(\log n)$ message-bits. It also follows that the number of small primes $k 2^{b}$ is polynomially bounded in $\log n$ so that, with $S^{\prime}$ the overly conservative RSA security level obtained according to the Computational 
VSSR Assumption, Fast VSH requires a single modular multiplication per $\Omega\left(S^{\prime}\right)$ message-bits.

Zero Chunks in Fast VSH. A negligible speed-up and tiny saving in the number of primes can be obtained in Fast VSH if for a particular $b$-bit pattern (such as all zeros) no prime is multiplied in (as was the case in basic VSH).

Fast VSH with Increased Block Length. Fast VSH can be used in a straightforward fashion with a larger block length than suggested by the above analysis. If, for instance, the number of small primes is taken almost $w$ times larger, for some integer $w>1$, the small prime product can be split into $w$ factors each less than $n$. Per iteration this results in a single modular squaring, $w-1$ modular multiplications plus the time to build the $w$ products. The best value for $w$ is best determined experimentally, and will depend on various processor characteristics (such as cache size to hold a potentially rather large table of primes).

Generating Collisions. For all variants given above knowledge of $\phi(n)$ can be used to generate collisions, though displaying such a collision is not in the user's interest since it would give out a break to the user's hash function (i.e. it would be similar to someone giving out the factorisation of their RSA modulus).

VSH-DL, a Discrete Logarithm Variant. We present a discrete logarithm (DL) variant of VSH that has no trapdoor. Its security depends on the following problem and its hardness assumption.

Definition 4. (VSDL: Very Smooth number Discrete Log) Let $p, q$ be primes with $p=2 q+1$ and let $k \leq(\log p)^{c}$. VSDL is the following problem: given $p$, find integers $e_{1}, e_{2}, \ldots, e_{k}$ such that $2^{e_{1}} \equiv \prod_{i=2}^{k} p_{i}^{e_{i}} \bmod p$ with $\left|e_{i}\right|<q$ for $i=1,2, \ldots, k$, and at least one of $e_{1}, e_{2}, \ldots, e_{k}$ is non-zero.

VSDL Assumption. The VSDL assumption is that there is no probabilistic polynomial (in $\log p$ ) time algorithm which solves VSDL with non-negligible probability (the probability is taken over the random choice of the prime $p$ and the random coins of the algorithm).

A solution to a VSDL instance produces the base 2 DL modulo $p$ of a very smooth number (the requirements on the exponents $e_{i}$ avoids trivial solutions in which all exponents are zero modulo $q$ ). Given $k$ random VSDL solutions, the base 2 DL of nearly all primes $p_{1}, \ldots, p_{k}$ can be solved with high probability by linear algebra modulo $q$. Although computing the DLs of a polynomial number of small primes is an impressive feat, it does not help to solve arbitrary DL problems. To solve the DL of an arbitrary group element with respect to some generator one could include both generator and element among the $p_{i}$, but there is no guarantee that solutions to VSDL contain the appropriate elements. Nevertheless, there is a strong connection between the hardness of VSDL and the hardness of computing DLs modulo $p$, which is reminiscent of, but seems to be somewhat weaker than, the connection between VSSR and factorisation. See also [3]. As was the case for VSSR, moduli for which VSDL is not difficult are easily constructed and not worthy of further consideration. 
Let $p$ be an $S$-bit prime of the form $2 q+1$ for prime $q$, let $k$ be a fixed integer length (number of small primes, typically $k \approx S / \log S$ ), and let $\mathcal{L} \leq S-2$. We define a VSH-DL compression function $H_{D L}:\{0,1\}^{\mathcal{L} k} \rightarrow\{0,1\}^{S}$, where $m$ is an $\mathcal{L} k$-bit message consisting of bits $m_{1}, m_{2} \ldots, m_{\mathcal{L} k}$ :

- Set $x_{0}=1$. For $j=0,1, \ldots, \mathcal{L}-1$, compute $x_{j+1}=x_{j}^{2} \times \prod_{i=1}^{k} p_{i}^{m_{j \cdot k+i}} \bmod p$.

- Return $H_{D L}(m)=x_{\mathcal{L}}$ interpreted as a value in $\{0,1\}^{S}$.

If $e_{i}=\sum_{j=0}^{\mathcal{L}-1} m_{j \cdot k+i} 2^{\mathcal{L}-j-1}$ for $1 \leq i \leq k$, then $H_{D L}(m)=\prod_{i=1}^{k} p_{i}^{e_{i}} \bmod p$. A collision $m, m^{\prime} \in\{0,1\}^{\mathcal{L} k}$ with $m \neq m^{\prime}$ therefore implies that $\prod_{i=1}^{k} p_{i}^{e_{i}} \equiv$ $\prod_{i=1}^{k} p_{i}^{e_{i}^{\prime}} \bmod p$, where $e_{i}^{\prime}=\sum_{j=0}^{\mathcal{L}-1} m_{j \cdot k+i}^{\prime} 2^{\mathcal{L}-j-1}$ and $m^{\prime}$ consists of the bits $m_{1}^{\prime}, \ldots, m_{\mathcal{L} k}^{\prime}$. Rearranging this congruence, a solution $2^{e_{1}-e_{1}^{\prime}} \equiv \prod_{i=2}^{k} p_{i}^{e_{i}^{\prime}-e_{i}} \bmod$ $p$ to VSDL follows, because $\left|e_{i}^{\prime}-e_{i}\right|<2^{\mathcal{L}} \leq 2^{S-2} \leq q$ for all $i$ and $e_{i}^{\prime}-e_{i} \neq 0$ for some $i$ since $m \neq m^{\prime}$. Hence the compression function $H_{D L}$ is collision-resistant under the VSDL assumption. We remark that VSH-DL can be viewed as a (more efficient) special case of the collision-resistant function in [3], which uses random group elements in place of the small primes $p_{i}$.

The compression function $H_{D L}$ uses the same iteration as the basic VSH algorithm. Hence, for the same modulus length $S$ and number of primes $k$ it has the same throughput efficiency of a single modular multiplication per about $\frac{k}{3}$ message-bits. By applying the Merkle-Damgård transformation $[15,8], H_{D L}$ can be used to hash messages of arbitrary length in blocks of $\mathcal{L} k-S$ message-bits per evaluation of $H_{D L}$. This leads to a reduction in throughput by a factor of $\frac{\mathcal{L} k-S}{\mathcal{L} k}$ (since only $\mathcal{L} k-S$ of the $\mathcal{L} k$ bits processed in each $H_{D L}$ evaluation are new message-bits) relative to factoring based VSH. However, for long messages, this throughput reduction factor can be made close to 1 by choosing a sufficiently large block length $\mathcal{L} k$; indeed, the construction allows block lengths up to $\mathcal{L} k=$ $k(S-2)$, and for this choice the throughput reduction factor is $1-\frac{S}{k(S-2)} \approx$ $1-\frac{1}{k} \approx 1$.

Reducing the Length. A possible drawback of VSH is its relatively large output length. We are investigating length-reduction possibilities by combining VSH-DL with elliptic curve, trace, or torus-based methods [10, 12, 22].

\section{VSH Randomised Trapdoor Hash and Applications}

Let $\mathcal{M}, \mathcal{R}, \mathcal{H}$ be a message, randomiser, and hash space, respectively. A randomised trapdoor hash function [20] $F_{p k}: \mathcal{M} \times \mathcal{R} \rightarrow \mathcal{H}$ is a collision-resistant function that can be efficiently evaluated using a public key $p k$, but for which certain randomly behaving collisions can be found given a secret trapdoor key $s k$ :

Collision Resistance in Message Input. Given $p k$, it is hard to find $m, m^{\prime} \in$ $\mathcal{M}$ and $r, r^{\prime} \in \mathcal{R}$ for which $m \neq m^{\prime}$ and $F_{p k}(m, r)=F_{p k}\left(m^{\prime}, r^{\prime}\right)$.

Random Trapdoor Collisions. There exists an efficient algorithm that given trapdoor $(s k, p k), m, m^{\prime} \in \mathcal{M}$ with $m \neq m^{\prime}$, and $r \in \mathcal{R}$, finds a randomiser 
$r^{\prime} \in \mathcal{R}$ such that $F_{p k}(m, r)=F_{p k}\left(m^{\prime}, r^{\prime}\right)$. Furthermore, if $r$ is chosen uniformly from $\mathcal{R}$ then $r^{\prime}$ is uniformly distributed in $\mathcal{R}$.

Randomised trapdoor hash functions have applications in provably strengthening the security of signature schemes [20], and constructing designated-verifier proofs/signature schemes $[11,21]$. The factorisation trapdoor of VSH suggests that it can be used to build such a function. Here we describe a provably secure randomised trapdoor hash family which preserves the efficiency of VSH.

Key Generation: Choose two $S / 2$-bit random primes $p, q$ with $p \equiv q \equiv 3 \bmod 4$ and $S$-bit product $n$. The public key is $n$ with trapdoor key $s k=(p, q)$. Let $k$ be as in the basic VSH algorithm, $\mathcal{M}=\cup_{\ell=0}^{2^{k}-1}\{0,1\}^{\ell}$, and $\mathcal{R}=\mathbf{Z}_{n}^{*}$.

Hash Function: Let $m \in \mathcal{M}$ of length $\ell<2^{k}$ and $r \in \mathcal{R}$. Calculate the basic VSH of $m$ with $x_{0}$ replaced by $r$ to compute $x_{\mathcal{L}+1}$ and output $F_{n}(m, r)=x_{\mathcal{L}+1}^{2} \bmod n$.

Theorem 2. The above construction satisfies the security requirements for randomised trapdoor hash functions, under the VSSR assumption.

Proof. Collision Resistance in Message Input: The proof follows the same lines as the proof of Theorem 1 since the value of $x_{0}$ and the squaring at the end do not affect the security reduction.

Random Trapdoor Collisions: Let $m, m^{\prime} \in \mathcal{M}$ with $m \neq m^{\prime}$ and $r \in \mathcal{R}$. Because $F_{n}(m, r)=\left(r^{2^{\mathcal{L}+1}} \prod_{i=1}^{k} p_{i}^{e_{i}}\right)^{2} \bmod n$, where $m_{\mathcal{L} k+i}=\ell_{i}$ and $e_{i}=$ $\sum_{j=0}^{\mathcal{L}} m_{j \cdot k+i} 2^{\mathcal{L}-j}$ for $1 \leq i \leq k$, finding $r^{\prime} \in \mathcal{R}$ with $F_{n}(m, r)=F_{n}\left(m^{\prime}, r^{\prime}\right)$ amounts to finding $r^{\prime}$ such that

$$
\left(r^{\prime}\right)^{2^{\mathcal{L}^{\prime}+2}} \equiv r^{2^{\mathcal{L}+2}} \cdot\left(\prod_{i=1}^{k} p_{i}^{e_{i}-e_{i}^{\prime}}\right)^{2} \bmod n
$$

(where $m_{\mathcal{L}^{\prime} k+i}^{\prime}=\ell_{i}^{\prime}$ and $e_{i}^{\prime}=\sum_{j=0}^{\mathcal{L}^{\prime}} m_{j \cdot k+i}^{\prime} 2^{\mathcal{L}^{\prime}-j}$ for $1 \leq i \leq k$ ), i.e., finding an $\left(\mathcal{L}^{\prime}+2\right)$ nd square root modulo $n$ of the right hand side $g$ of the equation for $\left(r^{\prime}\right)^{2^{\mathcal{L}^{\prime}+2}}$. Given the trapdoor key $(p, q)$ this is achieved as follows.

Let $Q R_{n}=\left\{y \in \mathbf{Z}_{n}^{*}:\left(\frac{y}{p}\right)=\left(\frac{y}{q}\right)=1\right\}$ denote the subgroup of quadratic residues of $\mathbf{Z}_{n}^{*}$. The choice $p \equiv q \equiv 3 \bmod 4$ implies that -1 is a quadratic nonresidue in $\mathbf{Z}_{p}^{*}$ and $\mathbf{Z}_{q}^{*}$, so for each element of $Q R_{n}$ exactly one of its 4 square roots in $\mathbf{Z}_{n}^{*}$ belongs to $Q R_{n}$. Hence the squaring map on $Q R_{n}$ permutes $Q R_{n}$ and given $(p, q)$ it can be efficiently inverted by computing the proper square roots modulo $p$ and $q$ and combining them by Chinese remaindering. Since $g \in Q R_{n}$, its $\left(\mathcal{L}^{\prime}+1\right)$ st square root $d \in Q R_{n}$ can thus be computed, and $r^{\prime}$ is then chosen uniformly at random among the 4 square roots in $\mathbf{Z}_{n}^{*}$ of $d$.

If $r$ is uniformly distributed in $\mathbf{Z}_{n}^{*}$, then (since each element of $Q R_{n}$ has 4 square roots in $\mathbf{Z}_{n}^{*}$ ) the value $r^{2} \bmod n$ is uniformly distributed in $Q R_{n}$. The squaring map on $Q R_{n}$ permutes $Q R_{n}$, so that $g$ and $d$ are also uniformly distributed in $Q R_{n}$. It follows that $r^{\prime}$ is uniformly distributed in $\mathbf{Z}_{n}^{*}$.

Efficiency. For short fixed-length messages with $\ell \leq k$ (i.e., 1 block), the message length can be omitted, so that $F_{n}(m, r)=\left(r^{2} \prod_{i=1}^{k} p_{i}^{m_{i}}\right)^{2} \bmod n$. Eval- 
uation requires only about 4 compared to at least $k$ modular multiplications required by the trapdoor functions in [20]. On the other hand, the trapdoor collision-finding algorithm for $F_{n}$ is not very fast, requiring a square root modulo $n$ per message block. This is not a major issue because in many applications of randomised hash functions, the collision-finding algorithm is only used in the security proof of a signature scheme rather than in the scheme itself. However, it reduces the efficiency of the reduction and thus requires slightly increased security parameters.

'Inversion' Trapdoor Property. It follows from the proof of Theorem 2 that $F_{n}$ also satisfies the 'inversion' trapdoor property [20]. This is stronger than the trapdoor collision property, and can be used to upgrade a signature scheme's resistance against random message attacks to chosen message attacks: Given the trapdoor key, a random element $d \in Q R_{n}$ in the range of $F_{n}$ and an $m \in \mathcal{M}$, it is easy to find a randomiser $r \in \mathbf{Z}_{n}^{*}$ such that $F_{n}(m, r)=d$ and $r$ is uniformly distributed in $\mathbf{Z}_{n}^{*}$ when $d$ is uniformly distributed in $Q R_{n}$.

Application. As an example application, we mention the Cramer-Shoup (CS) signature scheme [6], which to our knowledge is the most efficient factoring-based signature scheme provably secure in the standard model (under the strong-RSA assumption). The CS scheme makes use of an RSA-based randomised trapdoor hash function to achieve security against adaptive message attacks. Using $F_{n}$ instead cuts the signing and verification costs by about a double exponentiation each, while preserving the proven security. The modified CS scheme is as follows: Key Generation: Choose two safe random $\approx S / 2$-bit primes $\bar{p}, \bar{q}$ and two random $\approx S / 2$ bit primes $p, q$ with $p \equiv q \equiv 3 \bmod 4$ that result in $S$-bit moduli $\bar{n}=\bar{p} \bar{q}$ and $n=p q$, and choose $x, z \in Q R_{\bar{n}}$ at random. Let $h:\{0,1\}^{S} \rightarrow\{0,1\}^{\ell}$ be a collision-resistant hash function for a security parameter $\ell$ for which an $\ell$-bit (traditional) hash and $S$-bit RSA offer comparable security (typically $\ell=160$ when $S=1024)$. The public key is $(x, z, n, \bar{n}, h)$ and the secret key is $(\bar{p}, \bar{q})$. Signing: To sign $m \in\{0,1\}^{*}$, choose a random $(\ell+1)$-bit prime $e$ and a random $r \in \mathbf{Z}_{n}^{*}$ and compute $y=\left(x \cdot z^{h\left(F_{n}(m, r)\right)}\right)^{1 / e} \bmod \bar{n}$. The signature is $(e, y, r)$.

Verifying: To verify message/signature pair $(m,(e, y, r))$, check that $e$ is an odd $(\ell+1)$-bit integer and that $y^{e} z^{-h\left(F_{n}(m, r)\right)} \equiv x \bmod \bar{n}$.

The cost of verification in the original CS scheme is about two double exponentiations with $\ell$-bit exponents. The modified scheme requires approximately one such double exponentiation, so a saving in verification time of about $50 \%$ can be expected. The relative saving in signing time is smaller. However, the length of the public key is larger than in the original scheme by typically $25 \%$.

Because VSH's output length $S$ is typically much larger than $\ell$, VSH cannot be used for the $\ell$-bit collision-resistant hash function $h$ above. To avoid the need for an ad-hoc $\ell$-bit hash function, $h$ may be dropped and $e$ chosen as an $(S+1)$ bit prime, making the scheme much less efficient. The variant below eliminates the need for $h$ and maintains almost the computational efficiency of the scheme above, but has a larger public key and requires some precomputation. 
Key Generation: Let $\bar{p}, \bar{q}, p, q, \bar{n}, n$ be as above, let $s=\left\lceil\frac{S}{\ell}\right\rceil$ and randomly choose $x, z_{1}, \ldots, z_{s} \in Q R_{\bar{n}}$. The public key is $\left(x, z_{1}, \ldots, z_{s}, n, \bar{n}\right)$ with secret key $(\bar{p}, \bar{q})$.

Signing: To sign $m \in\{0,1\}^{*}$, choose a random $(\ell+1)$-bit prime $e$ and a random $r \in \mathbf{Z}_{n}^{*}$, and compute $F_{n}(m, r)$. Interpret $F_{n}(m, r)$ as a value in $\{0,1\}^{s \cdot \ell}$ (possibly after padding) consisting of $s$ consecutive $\ell$-bit blocks $F_{n, 1}(m, r), \ldots, F_{n, s}(m, r)$ and compute $y=\left(x \cdot \prod_{u=1}^{s} z_{u}^{F_{n, u}(m, r)}\right)^{1 / e} \bmod \bar{n}$. The signature is $(e, y, r)$.

Verifying: To verify message/signature pair $(m,(e, y, r))$, check that $e$ is an odd $(\ell+1)$-bit integer and that $y^{e} \prod_{u=1}^{s} z_{u}^{-F_{n, u}(m, r)} \equiv x \bmod \bar{n}$.

For typical parameter values such as $S=1024, \ell=171, s=6$, the $2^{s}=$ 64 subset products modulo $\bar{n}$ of the $z_{u}$ 's may be precomputed. Using multiexponentiation, that would make the above scheme about as efficient as the previous variant. It can be proved (cf. [4]) that the above CS signature variant is secure assuming the strong-RSA and VSSR assumptions. Thus we have obtained an efficient signature scheme proven secure without ad-hoc assumptions. This is unlike the original CS scheme, which relied on a collision resistance or universal one-wayness assumption regarding a 160-bit hash function - as far as we are aware, the only practical provably secure design for such a function is an inefficient discrete log based construction using an elliptic curve defined over a 160-bit order finite field. A disadvantage of our variant is that its public key is typically $9 \mathrm{kbits}$, which is about 3 times more than in the original CS scheme.

\section{Efficiency of VSH in Practice}

Let the cost of a multiplication modulo $n$ be $O\left((\log n)^{1+\epsilon}\right)$ operations, where $\epsilon=1$ if ordinary multiplication is used, and where $\epsilon>0$ can be made arbitrarily small if fast multiplication methods are used. Asymptotically the cost of the basic VSH algorithm is $O\left(\frac{(\log n)^{1+\epsilon}}{k}\right)=O\left((\log n)^{\epsilon} \log \log n\right)$ operations per message-bit. Given $n$ 's factorisation one can do better for long messages by reducing the $k$ exponents of the $p_{i}$ 's modulo $\phi(n)$. Asymptotically, Fast VSH costs $O\left((\log n)^{\epsilon}\right)$ operations per message-bit. It is faster in practice too, cf. below.

The table below lists VSH runtimes obtained using a gmp-based implementation on a $1 \mathrm{GHz}$ Pentium III. The two security levels conservatively correspond to 1024-bit and 2048-bit RSA (based on the Computational VSSR Assumption, where an $S$-bit VSH-modulus leads to a lower RSA security level $S^{\prime}$ depending on the number of small primes). In the 2nd and 6th rows basic VSH is used with more small primes, in the 3rd and 7th rows extended with precomputed prime products and message processing $b=8$ bits at a time. Fast VSH also processed $b=8$ message-bits at a time. With $S^{\prime}=1024$ and $S=1516$ (i.e., at least 1024-bit RSA security, at the cost of a 1516-bit VSH-modulus) Fast VSH is about 25 times slower than Wei Dai's SHA-1 benchmark [23]. Better throughput will be obtained under the more aggressive assumption that VSH with an $S$-bit modulus achieves $S$-bit RSA security. A similarly more favorable comparison will be obtained when using VSH with parameters matching the actual SHA-1 security level; at the time of writing that is 63 bits, but as it is a moving target 


\begin{tabular}{|c|c|c|c|c|c|c|}
\hline$S^{\prime}$ & Method & \# small primes & $S$ & $b$ & \# products & Megabyte/second \\
\hline \multirow{4}{*}{1024} & Basic VSH & 152 & 1234 & 1 & $\mathrm{n} / \mathrm{a}$ & 0.355 \\
\hline & & 1024 & 1318 & 1 & $\mathrm{n} / \mathrm{a}$ & 0.419 \\
\hline & & & & 8 & $128 * 256$ & 0.486 \\
\hline & Fast VSH & $2^{16}=65536$ & 1516 & 8 & $\mathrm{n} / \mathrm{a}$ & 1.135 \\
\hline \multirow[t]{4}{*}{2048} & Basic VSH & 272 & 2398 & 1 & $\mathrm{n} / \mathrm{a}$ & 0.216 \\
\hline & & 1024 & 2486 & 1 & $\mathrm{n} / \mathrm{a}$ & 0.270 \\
\hline & & & & 8 & $128 * 256$ & 0.303 \\
\hline & Fast VSH & $2^{18}=262144$ & 2874 & 8 & $\mathrm{n} / \mathrm{a}$ & 0.705 \\
\hline
\end{tabular}

we prefer not to specify matching VSH parameters. In any case, the slowdown is a small price for avoiding heuristically collision-resistant hashes. Nevertheless, except for its lack of other nice properties, VSH has been criticised for being too slow. We consider the prospects of faster VSH software more realistic than a proof that SHA-2 offers any security at all.

Acknowledgements. We gratefully acknowledge inspiring discussions with Igor Shparlinski and Eran Tromer, and we thank Yvo Desmedt, Josef Pieprzyk, Benne de Weger, and the anonymous Eurocrypt'06 reviewers for their insightful comments. This article was written while the second author was employed by Lucent Technologies' Bell Laboratories and was affiliated to the Technische Universiteit Eindhoven.

\section{References}

1. M. Bellare and D. Micciancio. A new paradigm for collision-free hashing: incrementality at reduced cost. In EUROCRYPT 97, volume 1233 of LNCS, page 163-192, Berlin, 1997, Springer-Verlag.

2. D. Boneh and M. Franklin. Efficient generation of shared RSA keys. In CRYPTO 97, volume 1294 of LNCS, page 425-439, Berlin, 1997, Springer-Verlag.

3. D. Chaum, E. van Heijst, and B. Pfitzmann. Cryptographically strong undeniable signatures, unconditionally secure for the signer. In CRYPTO 91, volume 576 of LNCS, page 470-484, Berlin, 1991, Springer-Verlag.

4. S. Contini, A.K. Lenstra, and R. Steinfeld. VSH, an efficient and provable collision resistant hash function. Report 2005/193, Cryptology ePrint Archive, 2005. eprint.iacr.org/2005/193/.

5. R. Crandall and C. Pomerance. Prime Numbers: a Computational Perspective, New York, 2001, Springer-Verlag.

6. R. Cramer and V. Shoup. Signature schemes based on the strong RSA assumption. In volume 3 of ACM Transactions on Information and System Security (ACM TISSEC), page 161-185, 2000.

7. I. Damgård. Collision-free hash functions and public key signature schemes. In EUROCRYPT 87, volume 304 of LNCS, page 203-216, Berlin, 1987, SpringerVerlag.

8. I. Damgård. A design principle for hash functions. In CRYPTO 89, volume 435 of LNCS, page 416-427, Berlin, 1989, Springer-Verlag.

9. S. Goldwasser, S. Micali, and R. Rivest. A digital signature scheme secure against adaptively chosen message attacks. SIAM J. on Comp., 17(2):281-308, 1988.

10. S. Hankerson, A. Menezes, S. Vanstone. Guide to Elliptic Curve Cryptography, New York, 2004, Springer-Verlag. 
11. M. Jakobsson, K. Sako, and R. Impagliazzo. Designated verifier proofs and their applications. In EUROCRYPT 96, volume 1070 of LNCS, page 143-154, Berlin, 1996, Springer-Verlag.

12. A.K. Lenstra and E.R. Verheul. The XTR public key system. In CRYPTO 2000, volume 1880 of LNCS, page 1-19, Berlin, 2000, Springer-Verlag.

13. A.K. Lenstra and H.W. Lenstra Jr. The Development of the Number Field Sieve, Berlin, 1993, Springer-Verlag.

14. A.K. Lenstra, E. Tromer, A. Shamir, W. Kortsmit, B. Dodson, J. Hughes, and P. Leyland, Factoring estimates for a 1024-bit RSA modulus. In Chi Sung Laih, editor, ASIACRYPT 2003, volume 2894 of LNCS, page 55-74, Berlin, 2003, SpringerVerlag.

15. R. Merkle. One way hash functions and DES. In CRYPTO 89, volume 435 of LNCS, page 428-446, Berlin, 1989, Springer-Verlag.

16. D. Naccache and J. Stern A new public-key cryptosystem. In Walter Fumy, editor, EUROCRYPT 97, volume 1233 of LNCS, page 27-36, Berlin, 1997, Springer-Verlag.

17. D. Pointcheval. The composite discrete logarithm and secure authentication. In PKC 2000, volume 1751 of LNCS, page 113-128, Berlin, 2000, Springer-Verlag.

18. R.L. Rivest and R.D. Silverman. Are 'strong' primes needed for RSA. Report 2001/007, Cryptology ePrint Archive, 2001. eprint.iacr.org/2001/007/.

19. P. Rogaway and T. Shrimpton. Cryptographic hash-function basics: definitions, implications, and separations for preimage resistance, second-preimage resistance, and collision resistance. In B. Roy and W. Meier, editors, FSE 2004, volume 3017 of LNCS, page 371-388, Berlin, 2004, Springer-Verlag.

20. A. Shamir and Y. Tauman. Improved online/offline signature schemes. In CRYPTO 2001, volume 2139 of LNCS, page 355-367, Berlin, 2001, Springer-Verlag.

21. R. Steinfeld, H. Wang, and J. Pieprzyk. Efficient extension of standard Schnorr/RSA signatures into universal designated-verifier signatures. In PKC 2004, volume 2947 of LNCS, page 86-100, Berlin, 2004, Springer-Verlag.

22. K. Rubin and A. Silverberg. Torus-based cryptography. In CRYPTO 2003, volume 2729 of LNCS, page 349-365, Berlin, 2003, Springer-Verlag.

23. Wei Dai. Crypto++ 5.2.1 Benchmarks. www.eskimo.com/ ${ }^{\sim}$ weidai/ benchmarks.html. 